\title{
TRANSFORMATIONS ASSOCIATED WITH THE LINES OF A CUBIC, QUADRATIC, OR LINEAR COMPLEX*
}

BY I. O. HORSFALL

1. Introduction. In this paper it is shown that two equations bilinear in $p_{i k}$ and $x_{i}$ define an extensive type of cubic complex and also map the complex on the space $(x)$ so that each line is mapped by a point on itself. The cubic complex of lines joining corresponding points of the general cubic involutorial transformation is included as a special case. The method is also applied to two known cases of the quadratic complex and the linear non-special and special complex.

2. The Cubic Complex. Let

$$
\sum x_{i} f_{i}(p)=0,
$$

and

$$
\sum x_{i} F_{i}(p)=0, \quad(i=1,2,3,4),
$$

be two equations bilinear in $x_{i}$ and the line coordinates

$$
p_{i k}=x_{i} y_{k}-x_{k} y_{i} .
$$

The $x_{i}$ and $p_{i k}$ satisfy four identities of the type

$$
x_{i} p_{j k}+x_{j} p_{k i}+x_{k} p_{i j}=0 .
$$

The equations (1) and (2) represent two quadrics through $(y)$ which meet in a $C_{4}$ through $(y)$. The lines of the cubic cone with vertex $(y)$ through $C_{4}$ belong to a cubic complex. If we eliminate the $x_{i}$ from (1) and (2) and any two of (3), we have the equation of the cubic complex each line $(l)$ of which is mapped by a point $(x)$ on $(l)$.

From (1), (2), and (3) we see that the $p_{i k}$ are quartic functions of $x_{i}$. Hence any linear complex meets the cubic complex in a cubic congruence which is mapped by a quartic surface $F_{4}(x)$. Two linear complexes meet the cubic complex in a cubic ruled

\footnotetext{
* Presented to the Society, October 29, 1932.
} 
surface which is mapped by the intersection of the two corresponding $F_{4}$ 's. Let

$$
x_{1}=x_{2}=0, \quad x_{3}=x_{4}=0
$$

be the pair of polar lines common to the linear complexes. If the coordinates of a line through a point $(x)$ meeting the polar lines are substituted in (1) and (2), we have two cubic surfaces through $x_{1}=x_{2}=0, x_{3}=x_{4}=0$ which meet in a residual $C_{7}$, the variable intersection of the two $F_{4}$ 's. The $C_{7}$ meets each polar line in 4 points and is of genus 4 . The common curve of all the $F_{4}$ 's meets $C_{7}$ in 22 points and is of genus 8 . Hence the cubic complex is mapped on $S_{3}$ by the linear system of quartic surfaces through a $C_{9}, p=8$.

3. The Cubic Complex of the General Cubic Involutorial Transformation. If $(y)$ and $(z)$ are conjugate points in the involutorial transformation they are polar conjugates with respect to three quadrics which by a suitable choice of coordinates have the equations*

Hence, if

$$
\begin{aligned}
& a_{1} x_{1} x_{4}+a_{2} x_{2} x_{4}+a_{3} x_{3} x_{4}-x_{2} x_{3}=0 \\
& b_{1} x_{1} x_{4}+b_{2} x_{2} x_{4}+b_{3} x_{3} x_{4}-x_{3} x_{1}=0 \\
& c_{1} x_{1} x_{4}+c_{2} x_{2} x_{4}+c_{3} x_{3} x_{4}-x_{1} x_{2}=0
\end{aligned}
$$

$$
g_{i j}=y_{i} z_{j}+y_{j} z_{i}, \quad(i, j=1, \cdots, 4),
$$

the three bilinear equations which define the involution may be written in the form

$$
\begin{gathered}
g_{23}=a_{1} g_{41}+a_{2} g_{42}+a_{3} g_{43}, g_{31}=b_{1} g_{41}+b_{2} g_{42}+b_{3} g_{43}, \\
g_{12}=c_{1} g_{41}+c_{2} g_{42}+c_{3} g_{43} .
\end{gathered}
$$

The $g_{i j}$ and $p_{i k}$ satisfy the identities which express the fact that $\left(g_{i 1}, g_{i 2}, g_{i 3}, g_{i 4}\right),(i=1, \cdots, 4)$, are on the line $p_{i k}$. Hence

(6) $g_{21} p_{34}+g_{23} p_{41}+g_{24} p_{13}=0, g_{12} p_{43}+g_{14} p_{32}+g_{13} p_{24}=0$.

If we substitute in (6) for $g_{23}, g_{31}, g_{12}$ from (5) and use $x_{i}$ for $g_{4 i}$, $(i=1,2,3,4)$, then (6) is the form of (1) and (2) for this case. These two equations with any two of (3) define the cubic com-

* F. R. Sharpe and V. Snyder, The $(1,2)$ correspondence associated with the cubic space involution of order two, Transactions of this Society, vol. 25 (1923), pp. 1-12. 
plex and map a line $(l)$ of the complex on a point $(x)$ of $(l){ }^{*}$ The cubic inversion $\nmid$ is the special case when

$$
a_{1}=b_{2}=c_{3}=1,
$$

and the other coefficients in (4) are zero.

4. The First Type of the Quadratic Complex. If the cubic complex of $\$ 2$ reduces to a quadratic complex, then $C_{4}$ must break up into a cubic curve through $(y)$ and either a line joining $(y)$ to a fixed point $O$, or a fixed line $(l)$.

In the first case the quadratic complex contains the bundle of lines through $O$, and the Kummer surface is a Steiner surface having three double lines through the triple point $O$. If the planes through the lines are

$$
x_{1}=0, \quad x_{2}=0, \quad x_{3}=0,
$$

the quadratic complex has an equation of the form

$$
a p_{23} p_{14}+b p_{31} p_{24}+c p_{12} p_{34}+F_{2}=0,
$$

where $F_{2}$ is quadratic in $p_{23}, p_{31}, p_{12}$.

Consider the bilinear equation

$$
p_{23}(A x)+p_{31}(B x)+p_{12}(C x)=0 .
$$

From equations (3), (7), and (8) we can derive the equations

$$
\frac{p_{23}}{x_{2}(C x)-x_{3}(B x)}=\frac{p_{31}}{x_{3}(A x)-x_{1}(C x)}=\frac{p_{12}}{x_{1}(B x)-x_{2}(A x)},
$$

which may be written

$$
\frac{p_{23}}{f_{1}}=\frac{p_{31}}{f_{2}}=\frac{p_{12}}{f_{3}}
$$

where $f_{i}=0$ is a quadric through a cubic $C_{3}$ which passes through $O$. Using (9) we find

* For another method of mapping the cubic complex see D. Montesano, Su di un complesso di rette del terzo grado, Bologna Memoria, 1893, pp. 549-577. See p. 565.

$\dagger \mathrm{L}$. Godeaux, Recherches sur les surfaces algébriques de genres zéro et de bigenre un, Académie Royale de Belgique, Classe des Sciences, Bulletin, (5), vol. 12 (1926), pp. 892-904. See pp. 896-897. 
1932.]

$$
\begin{aligned}
& p_{23}=f_{1}\left(a x_{1} f_{1}+b x_{2} f_{2}+c x_{3} f_{3}\right), \\
& p_{31}=f_{2}\left(a x_{1} f_{1}+b x_{2} f_{2}+c x_{3} f_{3}\right), \\
& p_{12}=f_{3}\left(a x_{1} f_{1}+b x_{2} f_{2}+c x_{3} f_{3}\right), \\
& p_{14}=x_{1} F_{2}(f)-(b-c) f_{2} f_{3} x_{4}, \\
& p_{24}=x_{2} F_{2}(f)-(c-a) f_{3} f_{1} x_{4}, \\
& p_{34}=x_{3} F_{2}(f)-(a-b) f_{1} f_{2} x_{4} .
\end{aligned}
$$

Hence if $(x)$ is given, the $p_{i k}$ are quintic functions of the $x_{i}$. Conversely, if the $p_{i k}$ are given satisfying (7), we can write (8) in the form

$$
d_{1} x_{1}+d_{2} x_{2}+d_{3} x_{3}+d_{4} x_{4}=0,
$$

and, using (3), we can find for $x_{i}$ the expressions

$$
x_{i}=d_{1} p_{i 1}+d_{2} p_{i 2}+d_{3} p_{i 3}+d_{4} p_{i 4}, \quad(i=1,2,3,4),
$$

which are quadratic in the $p_{i k}$. The quintic surfaces $(10), p_{i k}=0$, have $C_{3}$ for double curve. From (10) we see that if $p_{23}, p_{31}, p_{12}$ are fixed, then the lines of the complex lie in the fixed plane

$$
x_{1} f_{1}+x_{2} f_{2}+x_{3} f_{3}=0,
$$

and pass through the point $(y)$ on the Steiner surface, where

$$
\begin{array}{ll}
y_{1}=(b-c) f_{2} f_{3}, & y_{2}=(c-a) f_{3} f_{1}, \\
y_{3}=(a-b) f_{1} f_{2}, & y_{4}=F_{2}(f) .
\end{array}
$$

The equations (13) map the Steiner surface on a plane $\left(f_{1}, f_{2}\right.$, $f_{3}$ ). If we substitute the values of $(y)$ from (13) in (8) we have the condition that the point $(y)$ lies on the line in which (8) meets (12). This is a cubic relation in $(f)$ so that on the plane $(f)$ we have a cubic curve of genus 1 to which corresponds a $C_{6}, p=1$, on the Steiner surface meeting $C_{3}$ in the 9 points apart from $O$ in which $C_{3}$ meets the Steiner surface. This $C_{6}$ lies on all the quintic surfaces $p_{i k}=0$. The intersection of the quadratic complex with a linear complex is therefore mapped by a quintic surface $F_{5}: C_{3}^{2} C_{6}$. Two quintic surfaces meet in a variable $C_{7}$, $p=1$, meeting $C_{3}$ in 11 points and $C_{6}$ in 9 points. Three quintic surfaces meet in 4 variable points.

5. The Cremona Transformation Associated with the Quadratic Complex. Consider a second bilinear equation 


$$
\begin{aligned}
p_{23}\left(A^{\prime} x\right)+p_{31}\left(B^{\prime} x\right)+ & p_{12}\left(C^{\prime} x\right) \\
& =d_{1}^{\prime} x_{1}+d_{2}^{\prime} x_{2}+d_{3}^{\prime} x_{3}+d_{4}^{\prime} x_{4}=0 .
\end{aligned}
$$

Solving as in (11) for $x_{i}$ in terms of $p_{i k}$ and substituting from (10), we have expressions which are linear in the $F_{5}: C_{3}^{2} C_{6}$ with coefficients linear in $(f)$. Hence we have a Cremona transformation of order 7 of the form $F_{7}: C_{3}{ }^{3} C_{6}$. A surface $F_{7}: C_{3}{ }^{3}$ can be mapped on a plane by $C_{5}: 18 \mathrm{~A}$, quintics through 18 fixed points. The curve $C_{3}$ is mapped by $C_{9}: 18 A^{2}$ and the intersection of a variable $F_{7}: C_{3}{ }^{3}$ by $C_{8}: 18 A$. For the system $F_{7}: C_{3}{ }^{3} C_{6}$, the $C_{8}: 18 \mathrm{~A}$ consist of $C_{2}: 3 \mathrm{~A}$, image of the variable curve $C_{7}, p=0$, a fixed $C_{3}: 9 A$, image of $C_{6}, p=1$, and a fixed curve $C_{9}, p=1$. The triple curve $C_{3}$ meets $C_{6}$ and $C_{9}$ in 9 and 15 points, respectively, and $C_{7}$ in 12 points, $C_{6}$ and $C_{9}$ meet in 9 points and meet $C_{7}$ in 6 points.*

6. The Involutorial Transformation belonging to the Quadratic Complex. If we replace (14) by

$$
p_{23} H_{1}+p_{31} H_{2}+p_{12} H_{3}=0,
$$

where $H_{i}=0$ is a quadric, the surfaces $f_{i}=0$ are cubics through a $C_{7}, p=5$, which passes through $O$, and the surfaces $p_{i k}=0$ are of the form $F_{7}: C_{7}{ }^{2}$. The line in which the plane (8) met the plane (12) is replaced by a conic. If the point $(y)$ is on the conic, we have a relation of the fifth order in $f_{1}, f_{2}, f_{3}$ so that the surfaces $F_{7}$ are of the form $F_{7}: C_{7}{ }^{2} C_{10}, p=6$. The curve $C_{7}$ meets the Steiner surface in 25 points apart from $O$. Hence $C_{7}$ meets $C_{10}$ in 25 points. $\dagger$ To a line of the complex correspond two points $(x)$ on the line. Two surfaces $F_{7}: C_{7}{ }^{2} C_{10}$ meet in a variable $C_{11}, p=8$, meeting $C_{7}$ and $C_{10}$ in 27 and 15 points, respectively. Three of the surfaces meet in 8 variable points. Given a plane $(k x)=0$, we can find the $x_{i}$ in terms of the $k_{i}$ and $p_{i k}$ as in (11). Substituting these values for the $\left(x_{i}\right)$ in (15) and for the $p_{i k}$ from (10), we have a relation which is quadratic in the $k_{i}$ of which $(k x)$ is a factor. The other factor is the image of $(k x)=0$ in the involutorial transformation which interchanges the two points $(x)$

* Compare D. Montesano, Su le trasformazioni univoche dello spazio che determinano complessi quadratice di rette, Reale Istituto Lombardo Rendiconti, (2), vol. 25 (1892). See p. 803.

$\dagger$ Compare D. Montesano, Reale Istituto Lombardo Rendiconti, (2), vol. 25 (1892), p. 802. 
on a line $p_{i k}$ of the complex. This involutorial transformation is therefore of the form $F_{16}: C_{7}^{5} C_{10}{ }^{2}$. There are 25 trisecants of $C_{7}$ which meet $C_{10}$ so that the surfaces all contain these 25 parasitic lines.

7. The Second Type of the Quadratic Complex. If the quadrics (7) and (8) are replaced by

$$
a x_{1}=b x_{2}, c x_{1}=d x_{2},
$$

where $a, b, c$, and $d$ are linear in the $p_{i k}$, the lines belong to the quadratic complex $a d-b c=0$. The intersection of this complex with a linear complex is mapped by a quartic surface $F_{4}: l^{2}$, where $l \equiv x_{1}=x_{2}=0$. The intersection with a linear congruence is mapped by the intersection of two cubic surfaces through $l$ and the directrices of the congruence and is therefore a variable $C_{6}$, $p=1$, meeting $l$ in four points. Two of the surfaces $F_{4}: l^{2}$ meet therefore in a fixed $C_{6}, p=1$, meeting $l$ in four points. Consider a second pair of equations

$$
a x_{3}=b x_{4}, \quad c x_{3}=d x_{4},
$$

which give a second mapping of $a d-b c=0$. The two mappings determine a $(5,5)$ Cremona transformation $F_{5}: l^{3} C_{6}$. There is therefore an additional simple basis curve $C_{5}, p=0$, meeting $l$ and $C_{6}$ in four and eight points, respectively.*

8. The Second Type of Involutorial Transformation. If we replace (16) by

$$
a H_{1}=b H_{2}, \quad c H_{1}=d H_{2},
$$

where $H_{1}=0, H_{2}=0$ are quadrics meeting in $C_{4}, p=1$, the inter section of the quadratic complex $a d-b c=0$ with a linear complex is mapped by a sextic surface $F_{6}: C_{4}{ }^{2}$, and with a linear congruence by the intersection of two quartic surfaces through the directrices of the congruence and through $C_{4}$, that is, by a $C_{10}, p=7$, meeting $C_{4}$ in 16 points. Two surfaces $F_{6}: C_{4}{ }^{2}$ meet therefore in a variable $C_{10}, p=7$, and a fixed $C_{10}, p=7$, meeting $C_{4}$ in 16 points. On each line of the complex are two associated points $(x)$ and $\left(x^{\prime}\right)$. Given a plane $\Sigma a_{i} x_{i}^{\prime}=0$, we find

* F. R. Sharpe, Involutions of order $n$ with an $(n-2)$-fold line, Annals of Mathematics, (2), vol. 31 (1930), pp. 637-640. 


$$
x_{1}^{\prime}=a_{2} p_{12}+a_{3} p_{13}+a_{4} p_{14}
$$

and three similar equations. From (18) we have

$$
H_{1}^{\prime} H_{2}-H_{2}^{\prime} H_{1}=0 .
$$

Substituting for the $x_{i}^{\prime}$ and for the $p_{i k}$ in terms of the $x_{i}$ we have a relation which is quadratic in $a_{i}$ and has $\Sigma a_{i} x_{i}$ for a factor. The other factor is the image of $\Sigma a_{i} x_{i}^{\prime}=0$ by the involutorial transformation defined by (18). Hence the transformation is of the form $F_{13}: C_{4}^{5} C_{10}^{2}$. There are 16 bisecants of $C_{4}$ which are bisecants of $C_{10}$ and therefore lie on the $F_{13}{ }^{*}$

9. The Linear Complex. Consider the linear complex $p_{12}=p_{34}$ and the bilinear equation

$$
m_{2} p_{13}+m_{3} p_{14}+m_{4} p_{23}+m_{5} p_{24}+m_{6} p_{34}=0
$$

where the $m_{i}$ are linear in $x_{i}$. Proceeding as in $\$ 2$ we may show that the linear complex is mapped on $S_{3}$ by the linear system of cubic surfaces through a $C_{5}, p=1$.

The transformations belonging to a linear or special linear complex have been discussed synthetically by Montesano and Pieri. If we use one equation bilinear in $x_{i}$ and $p_{i k}$ and either $p_{12}=p_{34}$ or $p_{12}=0$, we can readily obtain the results of Montesanot and Pieri. $\ddagger$

\section{Cornell University}

* D. Montesano, Reale Istituto Lombardo Rendiconti, (2), vol. 25 (1892), p. 803.

$\dagger$ Compare D. Montesano, Napoli Accademia delle Scienze Fisiche e Matematiche, Rendiconti, (2), vol. 2 (1888), pp. 181-188. D. Montesano, Rendiconti dei Lincei, vol. 4 (1st semester), 1888, pp. 207-215, 277-285.

$\ddagger$ Pieri, Rendiconti di Palermo, vol. 6 (1892), pp. 234-244. 